investigated, and there needs to be a concerted effort to come to a consensus on what 'tolerance' to shift work means. Prospective studies would increase our understanding of which individual factors are associated with the development of tolerance over time, especially if they collect participants' complete occupational histories. Taking a paucity of evidence on these issues into account, the presentation will identify areas for future research with the goal of increasing evidence-based harm mitigation strategies for shift workers.

\section{S-319 NIGHT SHIFT WORK AND CANCER RISK: WHERE DO WE STAND, WHERE SHOULD WE GO?}

${ }^{1}$ Kyriaki Papantoniou. 'Medical University of Vienna, Austria

10.1136/OEM-2021-EPI.435

Introduction Night shift work was re-reclassified in 2019 by IARC/WHO as a probable human carcinogen (Group 2A) for humans, with limited epidemiological evidence for breast, prostate and colorectal cancer.

Objectives The objective of this talk is to provide an overview of the evidence on night shift work and cancer in epidemiological studies with a focus on breast cancer, to discuss strengths and limitations of existing studies and summarize areas for future research studies and policy actions.

Methods Among others, results from a pooled analysis of 5 population-based case-control studies of breast cancer using a common definition of night work (at least $3 \mathrm{~h}$ between midnight and 5 a.m.) will be presented. Results from a systematic Cochrane review on the effect of years of night shift work on cancer incidence will be summarized.

Results Women who ever worked at night had higher odds for breast cancer compared to never night workers (OR 1.12 $95 \%$ CI 1.00-1.25) in the pooled analysis. The risk was higher among pre-menopausal women (1.26; 1.06-1.51), high shift-work intensity and ER+ tumors. Our systematic review included 20 studies on breast cancer (12 case-control and 8 cohort studies). In preliminary meta-analysis, a non-linear dose-response relationship was found, with a $7 \%$ risk increase in breast cancer after 20 years of night work $(95 \%$ CI: 1.01-1.15). This finding was stronger in studies that reported lifetime occupational history and case-control studies.

Conclusions Night shift work of high intensity and long duration tends to increase the risk of breast cancer. Findings are stronger in studies with lifetime occupational history, among pre-menopausal women and positive hormone receptor subtypes. Other shift work research domains that need to be considered in future studies include 1) patterns of night work schedules 2) susceptible groups e.g. chronotype 3) critical exposure windows 4) co-exposures with occupational carcinogens.

\section{S-325 GENDERED OCCUPATIONAL INEQUALITIES AND HEALTH OVER LIFETIME: HOW CAN WE WORK THEM IN?}

${ }^{1} E$ Emilie Counil, Hyunji Byun, Mélanie Bertin. 'Institut national d'études démographiques (INED), France

10.1136/OEM-2021-EPI.436
Introduction Major economic and social changes occurred over the last 50 years, such as the increased participation of women in the labour market and the development of nonstandard forms of employment. These trends question the importance of work/employment conditions in the shaping of social inequalities in health over the life course. We thus hypothesize that (1) precarious occupational trajectories may contribute to ill health in adulthood, and that (2) they may affect genders differently.

Methods We used the French SIP (Santé Itinéraire Professionnel) national survey that collected information on occupational career and major health events of people aged 40-74 in 2006. We described poor employment conditions in terms of job instability, career discontinuity, qualification trends and versatility. We then applied multiple correspondence analysis and hierarchical ascending classification to identify patterns reflecting the accumulation of precarious employment conditions over job histories. Finally, we quantified the association between the type of occupational trajectory and self-reported health (Mini European Health Module) through multivariate logistic regression.

Results We included more than 9500 participants of workingage or $<5$-years retirees at the time of the survey. The classification showed that women were over-represented among most precarious trajectories. We also found that people with precarious trajectories more often reported less than good selfperceived health, currently experiencing longstanding illness or health problem, and activity limitation due to health problems. The trend of increasing ill health across work trajectories (stable/qualified/continuous trajectories serving as a reference) was similar among men and women, although adjusted ORs were slightly higher among women.

Conclusion We bring evidence that women experienced more precarious employment trajectories in France over the last decades than did men, with similar health outcomes among men and women. We now seek to expand our gendered perspective by taking into account hazardous working conditions as a potential mediating pathway.

\section{S-337 EXPOSURE TO PESTICIDES AND CANCER OF THE LYMPHOHEMATOPOIETIC SYSTEM IN THE AGRICOH COHORT CONSORTIUM}

${ }^{1}$ Karl-Christian Nordby, on behalf of AGRICOH Working Group on Cancer. ${ }^{1}$ National Institute of Occupational Health, STAMI, Norway

\subsection{6/OEM-2021-EPI.437}

Objective Pesticide exposure has been associated with certain cancer outcomes among farmers and applicators spraying pesticides. In the AGRICOH consortium of agricultural populations, we have evaluated cancer risk and pesticide exposure in three large cohort studies: The French Agriculture and Cancer Study - AGRICAN (FR), Agricultural Health Study (US), and Cancer in the Norwegian Agricultural Population (NO) studies.

Methods Estimates of lymphohaematologic cancers from the three cohorts (FR, US and NO) were analysed individually and then meta-analysed to yield more robust estimates of associations. Ever-never exposure to 33 chemical active ingredients from 14 chemical groups of agrochemicals was assessed using questionnaire information (US) and Crop Exposure 